\title{
NOVEL SENSOR TECHNOLOGY INTEGRATION FOR OUTCOME-BASED RISK ANALYSIS IN DIABETES
}

\author{
Mahesh Subramanian $^{1}$, Edward C.Conley ${ }^{1,2}$, Omer F.Rana ${ }^{1}$, Alex Hardisty ${ }^{1}$, Ali Shaikh Ali $^{1}$, \\ Stephen Luzio ${ }^{2}$, David R Owens ${ }^{2}$ \\ ${ }^{1}$ The Welsh e-Science Centre, Cardiff University School of Computer Science, \\ 5 The Parade, Roath, Cardiff, UK, CF24 3AA and \\ ${ }^{2}$ Diabetes Research Unit, Cardiff University School of Medicine, \\ Academic Centre, Llandough Hospital, Penlan Road, Penarth, Cardiff CF64 2XX \\ \{m.subramanian, e.c.conley, o.f.rana, alex.hardisty, ali.shaikhali\}@cs.cf.ac.uk; \{luzio, owensdr\}@cf.ac.uk
}

Steve Wright, Tim Donovan, Bharat Bedi, Dave Conway-Jones, David Vyvyan, Gillian Arnold IBM United Kingdom Ltd, Hursley House, Hursley Park, Winchester, UK, SO21 2JN

\{steve_wright, tim_donovan, bharat_bedi, conway,drvyvyan, arnoldg\}@uk.ibm.com

Chris Creasey, Adrian Horgan, Tristram Cox

Smart Holograms, 291 Cambridge Science Park, Milton Road, Milton, Cambridge, UK, CB4 OWF

\{chris.creasey, adrian.horgan, tristram.cox\}@smartholograms.com

and Rhys Waite

Zarlink Semiconductor Ltd, Phase 2 Mitel Business Park, Caldicot, Monmouthshire,UK, CF26 5YW

rhys.waite@zarlink.com

Keywords: Health informatics, home healthcare, biomedical sensor devices, mobility, wearable sensors, decision support system, individualised risk analysis.

Abstract: Novel sensor-based continuous biomedical monitoring technologies have a major role in chronic disease management for early detection and prevention of known adverse trends. In the future, a diversity of physiological, biochemical and mechanical sensing principles will be available through sensor device 'ecosystems'. In anticipation of these sensor-based ecosystems, we have developed Healthcare@Home $(\mathrm{HH})$ - a research-phase generic intervention-outcome monitoring framework. $\mathrm{HH}$ incorporates a closedloop intervention effect analysis engine to evaluate the relevance of measured (sensor) input variables to system-defined outcomes. $\mathrm{HH}$ offers real-world sensor type validation by evaluating the degree to which sensor-derived variables are relevant to the predicted outcome. This 'index of relevance' is essential where clinical decision support applications depend on sensor inputs. $H H$ can help determine system-integrated cost-utility ratios of bespoke sensor families within defined applications - taking into account critical factors like device robustness / reliability / reproducibility, mobility / interoperability, authentication / security and scalability / usability. Through examples of hardware / software technologies incorporated in the $\mathrm{HH}$ end-to-end monitoring system, this paper discusses aspects of novel sensor technology integration for outcome-based risk analysis in diabetes.

\section{INTRODUCTION}

Continuous sensor-based monitoring technologies are central to new models of 'proactive' health and social care. In healthcare, 'proactive' implies a shift away from 'reactive' care - i.e. an 'illness-centric' model where interventions are made following presentation of symptoms or complications. The 'proactive' model embraces the World Health Organisation's (WHO) definition of health as " $a$ state of complete physical, mental and social wellbeing and not merely the absence of disease or infirmity" (WHO, 2007). To move towards this 
visionary goal, individuals need realistic opportunities to improve and sustain their health and quality of life thus contributing to their own wellbeing. The availability of validated, relevant and ubiquitous personal healthcare information to minimise risk of predictable adverse events can empower and incentivise individuals to adopt more healthy lifestyles. Such technology can assist care throughout the 'patient path' (Abidi, 2001; Shnayder, 2005). Arising from these technology developments are significant ethical issues - e.g. in personal data protection and in establishing ethical authority for personal data reuse. We will discuss these issues in detail elsewhere in the context of our Healthcare@Home $(\mathrm{HH})$ project - a research-phase generic intervention-outcome monitoring framework that integrates sensor-based technology as part of a disease early detection and prevention framework.

It is widely anticipated that future health information systems (HIS) will need to move from "institution-based" models to those that rely on 'near real time' data integration close to the patient. Interventions that use ethical risk stratification as part of a personal data integration framework is a priority in diabetes, where the number of affected individuals is predicted to rise from c.135 million people in 1995 to c.300 million in 2025 (King, 1998). All people with unmanaged diabetes are at substantially increased risk of serious medical complications such as retinopathy, kidney failure and peripheral neuropathy requiring limb amputation. As part of an individual's personal information management, the $H H$ closed-loop model uses sensor-based trends to compute and stratify risk in a time frame and operational workflow that is meaningful and in a format that can be utilised for building decision support services (DSS). The DSS model in $\mathrm{HH}$ is founded on requirements of the Diabetes National Service Framework (NSF) standards for Wales and associated integrated care pathways (ICP). Section 2 summarises relevant related work. Section 3 describes technical aspects of the end-to-end $H H$ system covering (1) smart sensors; (2) biometric authentication; (3) 'home hub' and (4) server-side architecture. Section 4 discusses concerns arising out of the project and possible future work with a conclusion in Section 5.

\section{RELATED WORK}

A healthcare technology platform utilising sensor devices can underpin comprehensive monitoring services outside of the hospital environment. This could support new ways of working that: (1) places less reliance on frequent clinical visits - subject to quality control / calibration safeguards and adequate clinical 'baseline' data (2) can incentivise patients to 'look after themselves' with realistic (achievable) personal guidelines within manageable episodes of care; (3) allows team-based caseload sharing between clinical visits to monitor progress and make escalation procedures robust (4) provides for the development of consistent risk prediction / longitudinal 'outcome recording' methodologies that are fit-for-purpose in scaleable evidence-based models (Williams, 2003; Conley, 2007). Several research projects address the issue of integrated care through the use of ubiquitous computing devices. SAPHIRE (Hein 2006) is concerned with developing a healthcare monitoring and decision support system for cardiovascular disorders, assisted by wireless sensor devices in home settings. (Clemensen 2004) applied pervasive computing devices to the treatment / monitoring of diabetic foot ulcers. The CODEBLUE project (Lorincz, 2004) is typical of sensor device applications in medical emergency scenarios. In this project, micro-scale sensor devices (motes) (Crossbow, 2007) have been used to continuously monitor and wirelessly transmit vital sign data (e.g. heart rate and oxygen saturation data) to a data hub for processing. The CART (continuous automated real-time triage) system, developed by Advanced Health and Disaster Aid Network (AID-N) builds on the work carried out by the CODEBLUE team. A wearable tag has been developed which performs the following functions: triage, status display, vital signs monitoring, location tracking, information display and alarm signalling (Gao, 2006). The Smart and Aware Pervasive Healthcare Environment (SAPHE, 2007) supports telecare and lifestyle monitoring paradigms for early detection and prevention of adverse events i.e. for intervention before they become critical or lifethreatening. Several remote healthcare monitoring systems are that currently use proprietary device information systems (e.g. Honeywell, American Telecare and AMD Telemedicine). Many more device families are expected to be developed in coming years conforming to global standards being established by the Continua Healthcare Alliance companies (Continua, 2007).

The $H H$ system's conceptual driver is enabling 'near real time' risk analysis for early detection and prevention of disease. A Web Services-based platform to 'push' or 'pull' individual's healthrelated data along the patient path is being configured in a manner that will reduce transcriptional errors. The end-to-end framework 
(Figure 1, see below) employs a collection of clinical hubs, mobile devices and / or dedicated home-based network servers to one or more data analysis engines.

\section{END-TO-END FRAMEWORK}

Figure 1 illustrates the conceptual design of an 'endto-end' framework adopted in the $\mathrm{HH}$ project. The framework allows data capture from both wearable sensors and specialist hand-held instruments with wireless data transfer capability. Through a messaging fabric and / or dedicated integration application, raw data used in the risk analysis modules may originate from a wide variety of sources and device types (e.g. electronic forms, physiological monitors, retinopathy cameras, scanners, clinical chemistry or nucleic acid sequencing instrumentation). The data is integrated using a schema indicated as "QUID” (QUantitative Individualised Data integration) in the figure. In the context of an 'initial assessment' workflow, the types of diagnostic test to be performed are specified by a clinical registration procedure to be described elsewhere. The registration procedure has specific functions to ensure compliance to ethical protocols and informed consent procedures for re-use of data while verifying patient identity (see below). $\mathrm{HH}$ has adopted the Diabetes Continuing Care Reference (DCCR) dataset as the basis of its schema).
Subsequent risk analysis of baseline data is undertaken in the context of a "disease model" - a quantitative research-based predictive framework that indicates which risk variables are most relevant to system-specified outcomes. In this regard, $\mathrm{HH}$ has been conceived as a comprehensive healthcare outcomes evidence-based learning platform.

\subsection{Smart sensors}

In monitoring applications, the most common physiological analytes are blood gases (e.g. carbon dioxide, oxygen), blood electrolytes (e.g. potassium, sodium, and chloride), blood glucose, creatinine, urea, $\mathrm{pH}$, cholesterol, bilirubin and proteins (e.g. albumin). The relative significance of values depends on the type of investigative scenario and progression status of disease. In diabetes, the measurement of glucose concentration at an appropriate frequency is routine in short-term prevention of hyper- and hypo-glycaemic events and in long-term prevention of complications. Automated measurements can be enabled to support continuous monitoring. Conventionally, glucose monitoring is performed using 'finger-stick' devices. These provide only intermittent measurements and rely on patient compliance. Next-generation glucose monitoring devices aim to provide real-time continuous measurements by non- or minimallyinvasive means. One such sensor designed for integration into the $H H$ system uses glucoseresponsive holograms based on thin-film polymers

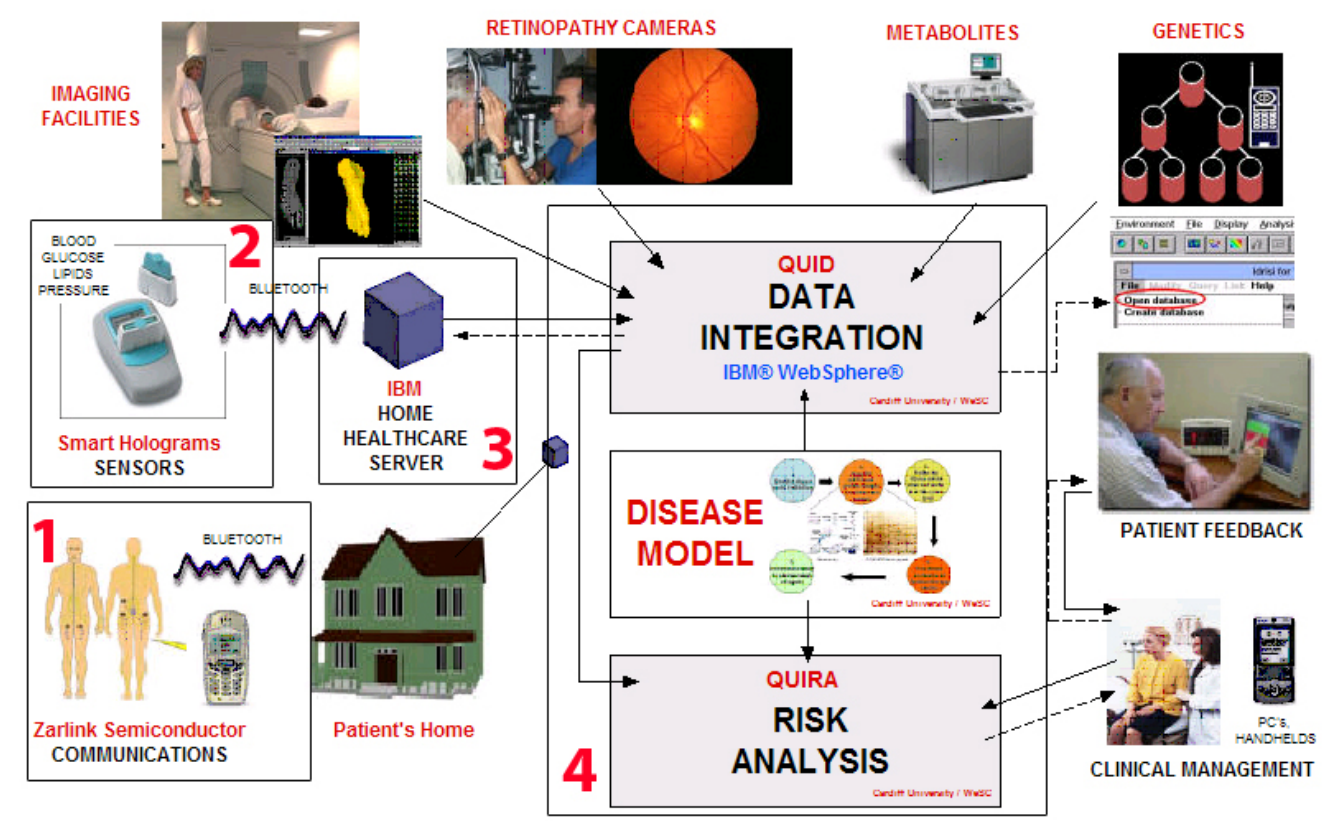

Figure 1: $H H$ end-to-end framework 
incorporating phenylboronic acid receptors. Selective binding of glucose to the receptors induces swelling or contraction of the film. This physical change in turn causes the spacing between holographic fringes within the film to increase or decrease, thereby modulating the colour of the light diffracted according to Bragg's Law.

To obtain repeatable quantitative measurements (figure 2), the colour of the hologram can be read with a portable optical reader equipped with Bluetooth ${ }^{\circledR}$. These sensors exhibit long-term chemical and physical stability, enabling measurements over long time periods without evidence of hysteresis. A further advantage is the ability of holographic analyte sensors to be incorporated into a multitude of formats (e.g. catheters, contact lenses, implants), thereby offering increased patient choice.

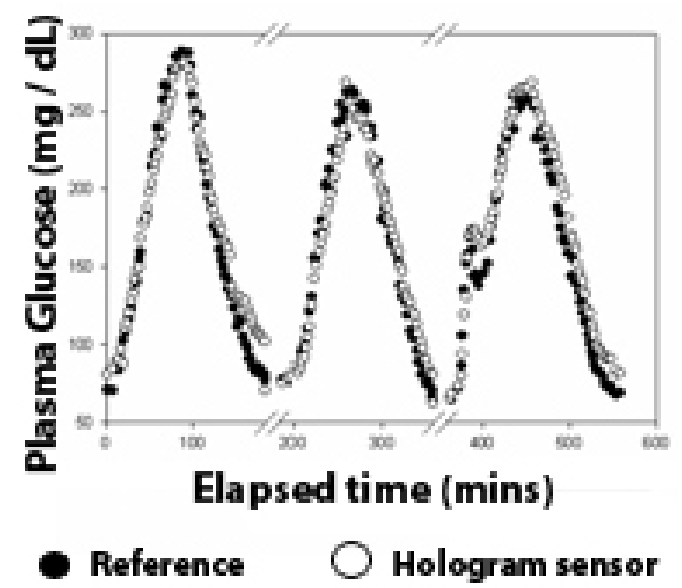

Figure 2: Real-time measurement of blood glucose using a holographic sensor compared to measurements made off-line and post experiment by a traditional reference method.

The prototype hand-held device (figure 3 ) has a touch-screen user interface. The reader employs a bespoke spectrometer optical sub-assembly, combined with analogue to digital converters (ADCs). These ADCs are read by a microprocessor which provides a calibrated output of wavelength and the corresponding concentration of the analyte being measured. This is achieved by use of look up tables or calibrated polynomial curve fit data. Temporal data trends can be calculated on-device or data can be sent via Bluetooth ${ }^{\circledR}$ to a $H H$ web service to enable downstream decision support.

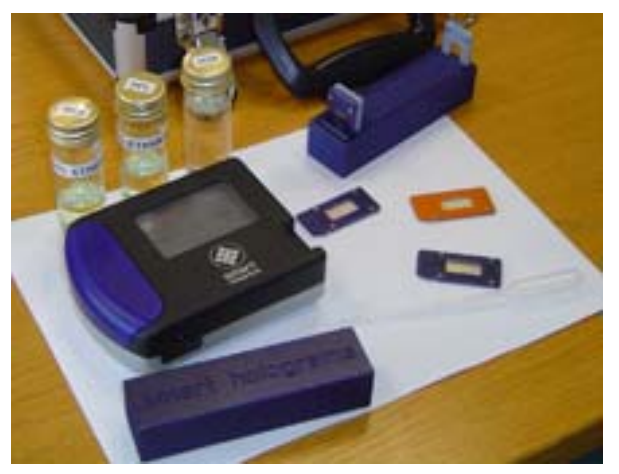

Figure 3: Smart Holograms prototype Hologram reader with integrated Bluetooth ${ }^{\circledR}$ connectivity.

\subsection{Biometric authentication}

The $H H$ system design has evaluated scenarios where people entering data might be easily confused by similar names or as different members of the same family. A number of protocols for disambiguation and absolute patient identification can be incorporated, including biometrics and / or smartcards. Biometric variables can be classified either as physiological (e.g., derived from a fingerprint, face or iris scan) or behavioural (e.g., speech recognition) (Biometrics, 2007).

The $H H$ system requires technology options that are cost-effective, fast and accurate. Based on these criteria, biometric identification used robust fingerprint recognition technology (' 2 ' in figure 1) (Wilson 2003). For flexibility, the biometrics device was designed to be "loosely-coupled" with the sensor and the home hub modules (refer to section 3.3). This permitted different packaging options with integration of new sensor types without substantial additional engineering cost. The hub is used to tag the biometric ID to the incoming sensor data in order to form an association between a patient identity and a data reading. Communication between sensor, home hub and the biometrics module is via Bluetooth ${ }^{\circledR}$, using a protocol specified by IBM, Zarlink and Smart Holograms.

In its current operational mode, the biometric device (figure 4) used in the $H H$ system saves the scanned fingerprint of a user in local memory, and the individual is prompted to assign an "ID" specific to that fingerprint. That ID is then transmitted via Bluetooth ${ }^{\circledR}$ to the data hub to be tagged within an electronic patient record. This “enrolment” process can be used prior to sensor readings to validate identity. A delay-free smartcard that has high enduser acceptance (e.g. contact-less 'wave \& pay' 
cards) can also be used in appropriate circumstances e.g. to enable inter-service access along the patient path. Identification of the patient by means of their enrolled ID sends that ID to the patient-proximal hub (in the clinical data 'baseline data' operational hub or the patient's own home hub, or mobile hub). The home hub can associate the subsequent sensor reading with the patient ID. This is the "identification" process. The design of the device ensures security of patient information. All scanned fingerprints are stored in the memory of the biometric device. No fingerprint scans are transmitted. All patient-identifiable information can be encrypted at source, in transmission and storage. If a hacker were to capture the wireless transmissions the data would have no meaning.

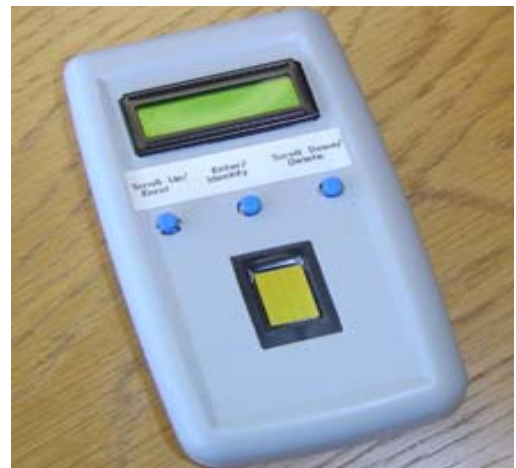

Figure 4: Fingerprint scanner by Zarlink

\subsection{Home Hub}

The function of the 'home hub' (' 3 ' in figure 1) is to collect and collate the data from sensor(s) and the biometric and smartcard / reader device(s) and to cotransmit these via an appropriate communication channel to the remotely-located server. The home hub sits at the centre of the data collection and transmission capability of the system, where all devices - sensors, authentication module and server connect through common interfaces. Various physical realizations of the hub are possible. It can, for example, be a mobile device such as a standard mobile phone (we have demonstrated functionality on a Sony Ericsson P910 phone - figure 5) that can provide near real-time data connectivity. The hub can also be deployed as a fixed 'wired' hub using, for example, Ethernet connectivity. In a clinical environment this also provides near real-time data connectivity. For home use, hubs can be configured to upload data periodically e.g. once or twice a day.

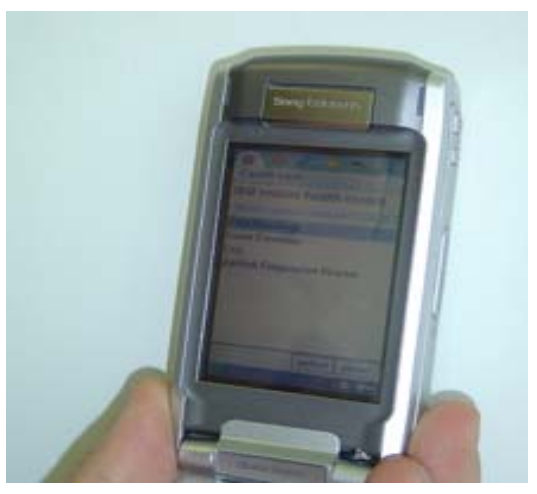

Figure 5: Mobile phone personal data hub by IBM

Sensor devices currently connect to the hub through adapters specific to the type of interface required. Adapters are device transmission protocol specific and can be developed by any manufacturer wanting to provide connectivity of their devices to this infrastructure. In $H H$, the medical devices use Bluetooth ${ }^{\circledR}$, although the architecture allows for this to be any available. Zigbee has some power consumption advantages over Bluetooth ${ }^{\circledR}$ (Zigbee, 2007).

We have developed adapters for a variety of devices that can be used in conjunction with the demonstrator system; namely for: (1) weighing scales; (2) blood pressure cuff; (3) pulse oximeter; (4) glucose meter. In addition to adapters for sensor devices, an adapter is also required to interface the hub to an appropriate application server. This step is also protocol-specific and in practice a range of adapters may be needed according to specific application scenarios.

The hub architecture (figure 6) consists of an event engine that behaves much like a broker, in that it can receive events from one adapter and passes these onto another adapter. In the simplest operation, the event engine receives events from a single sensor device (for example, weighing scales) and sends this event to the adapter that transmits these events to an application server. The current implementation of this architecture uses the IBM Personal Care Connect toolkit (Blount, 2007) and is based around a number of standard technologies. These include:

- Java 2, Micro Edition, Mobile Information Device Profile, Connection-Limited Device Configuration (J2ME, MIDP, CLDC) or OSGI for fixed hub (Java ME, 2007).

- $\quad$ JSR-82 to allow Java to interface to Bluetooth ${ }^{\circledR}$ (assuming Bluetooth-enabled medical devices) (JSR 82, 2007). 
- $\quad$ Bluetooth ${ }^{\circledR}$ or mobile connectivity via GSM / GPRS for wireless hubs.

- $\quad$ Ethernet, ISDN, etc. for fixed / wired hubs.

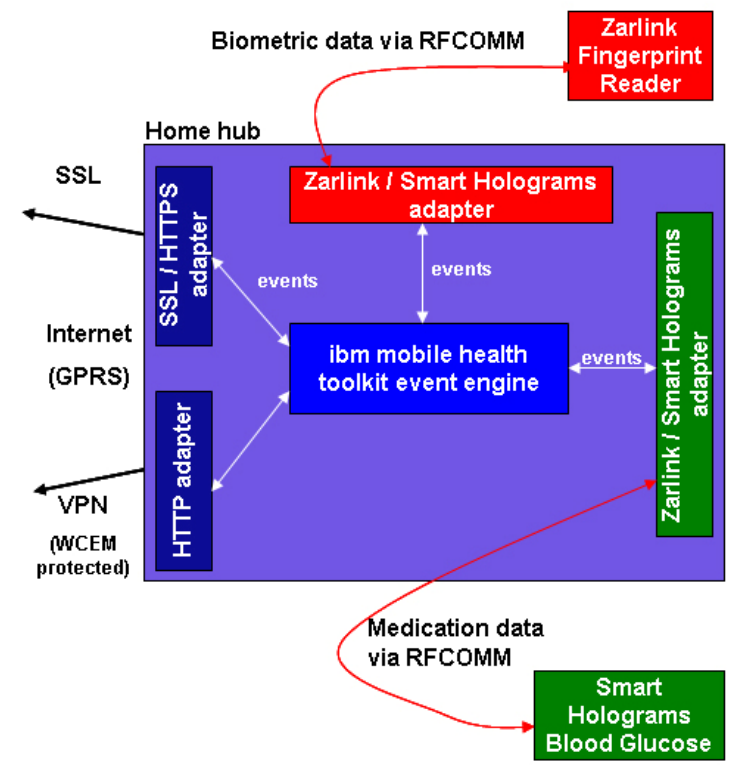

Figure 6: Hub technical overview

The hub design supports multiple adapters - key to creation of an open-hub platform. Each adapter is specific to a hub family, which is characterised by the following attributes: (i) device-to-hub communication, (ii) event data representation, (iii) event serialisation, and (iv) hub-to-server interaction. Different hub families can use different event classes, communication protocols, and serialisation schemes. A hub family may be optimised to handle a specific set of biomedical sensor types. Each adapter handles communication with a family of hubs by (i) defining a protocol, (ii) de-serialising the data forwarded by the hub, and (iii) extracting event data from the event after instantiating the events. Adapters also convert proprietary data events into a consistent format - i.e. act as a definable interface. It is expected that $\mathrm{HH}$ will migrate to industry recommendations of consortia such as the Continua Alliance (Continua, 2007). The hub functionality is currently implemented in Java and is deployable to a mobile phone as a MID-let or to a fixed hub as a set of OSGI bundles.

In the current research demonstrator, sensor data can be obtained from devices and stored on the hub prior to transfer by a standard HTTP protocol. A HTTP 'Post' agent uses stable storage to cache events that cannot be sent immediately and then forwards them when the next connection to the server is established. Data sent to the server is secured using the Secure Sockets Layer (now referred to as Transport Layer Security) (SSL, TLS). Other options include: Access Point Node (APN) and Virtual Private Network (VPN).

Secure Sockets Layer: SSL is commonly used within internet applications to provide secure client to web server connections.

Access Point Node: The Access Point Node (or Name) is the definition of the internet connection on a GPRS mobile device that provides the route the data will take from the GPRS device to access other networks such as the Internet. All APN's are defined within the mobile device with a username and password. The APN concept assumes the existence of GPRS support nodes that exist between a mobile device (using a GSM or UMTS service) and a server providing IP-based access to the Internet. By obtaining a private APN (with its own unique credentials) it is possible to create a private connection back to the $H H$ servers. The $H H$ demonstrator has been successfully tested with APN.

Virtual Private Network: A VPN effectively creates a private network by creating a secure 'tunnel' through the existing IP network. The $H H$ demonstrator has successfully used the IBM VPN product WebSphere Everyplace Connection Manager (WECM).

A sub-class of specialised functions that mimic adapters exist to create functions internal to the hub. A number of these have been implemented within the current demonstrator. For example, an "Audio Alert Agent" makes pre-configured audio alerts in response to certain events like the reception of data from a device or successful transmission of an event to the server. The "User Display Agent" enables the display of status information and provides input capability for the user.

\subsection{Server-side architecture}

The server-side architecture comprises of a 'QUID' and 'QUIRA' component (figure 7 and ' 4 ' in figure 1 ) and has been described in detail elsewhere (Subramanian, 2006, Shaikh-Ali, 2007, Conley, 2007).

The QUID component focuses on delivering data collection, data storage, process execution and portal infrastructures to support users of the system including clinicians, patients and researcher roles. The development of QUID has been guided by the 


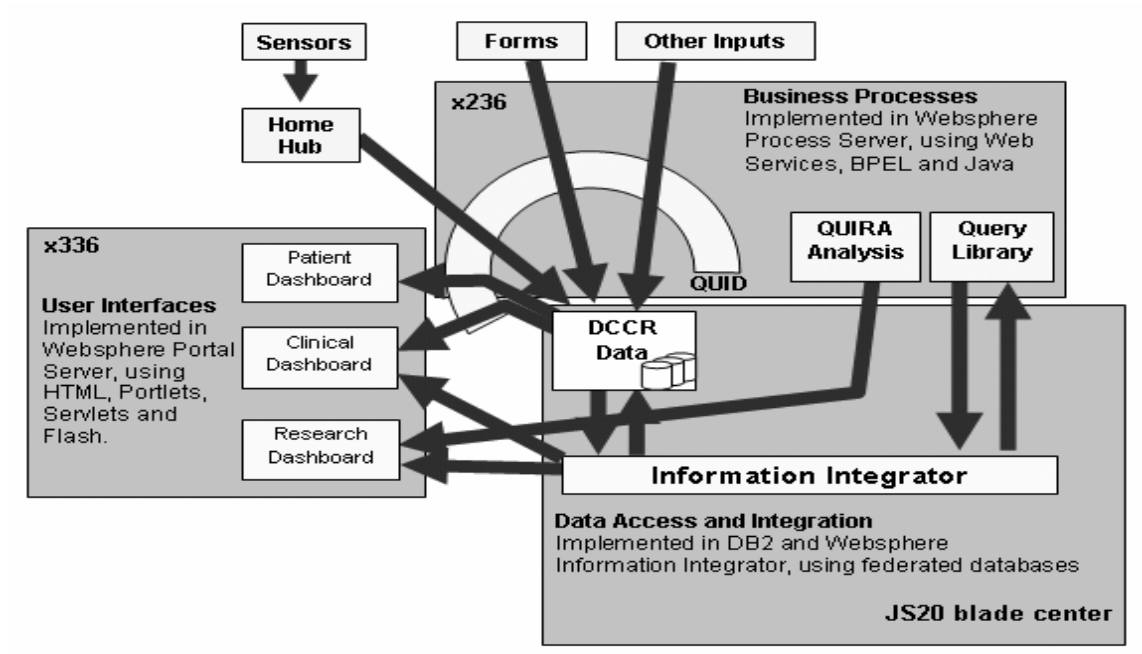

Figure 7: Physical implementation of the conceptual design shown in Figure1

requirements of Integrated Care Pathways (ICPs) for diabetes (ICP, 2007). The QUID component collects, validates and stores data streams from hubs along the patient path. The interface for data presentation / collection emulates workflows in realworld care pathways. This 'end-user familiarity' design feature is strengthened by presentation of the sensor data in a timeline-based (longitudinal) layered manner, make the data meaningful. We have used OpenLaszlo (Laszlo, 2007) to display charts in an intuitive manner. Similarly, the patient portal permits access to personal data supporting the self-management paradigm

The QUIRA (Quantitative Individualised Risk Analysis) component comprises a risk analysis engine performing various operations identifying signals in the longitudinal data stream, alerting the care team to fulfilment of pre-defined risk criteria. QUIRA represents ongoing research we will report on in the future.

\section{CONCERNS \& FUTURE WORK}

"Pervasive" or "ubiquitous" computing covers a range of research topics, including distributed computing, mobile computing, sensor networks, communications, artificial intelligence, and humancomputer interaction. It is an emerging field of research, and as such has many unresolved issues notably in areas like security, usability, privacy and ethics amongst others. In the framework described in this paper, ubiquitous computing devices demand secure transmission of data to the server, in turn demanding encryption mechanisms that defeat purposeful or accidental 'eavesdropping'. Device miniaturisation assists the resolution of powering issues. Significant ethical and privacy issues remain, and it is axiomatic that a comprehensive informed consenting process needs to be developed that is fitfor-the defined purpose of disease early detection and prevention. Informed consent needs to be properly structured in the recording workflow.

There is currently no widely-accepted standard protocol for the device-to-server data transmission and / or format / structure for data being transferred between devices. We expect interoperability between devices from different vendors to be a key focus within the scope of the Continua Healthcare Alliance (Continua, 2007). Irrespective of global technical standards adopted, methodological standardisation of data acquisition needs to be defined in order for patients and carers to reap the benefits of interoperable systems.

The $H H$ project has to date employed noninvasive sensors. However we anticipate significant developments in coming years in the area of invasive (implantable) sensors. Implantable sensors will likely be micro-miniaturised devices that can be implanted into a patient's body to enable relaying of health-critical signals on a semi-continuous basis.

\section{CONCLUSION}

System-based management of chronic conditions is essential to improve healthcare outcomes. Conventional models of healthcare provision lack capacity to continuously monitor physiological data 
combined with life event 'timelining'. In such an information system, healthcare can be provided in a 'patient-centric' model that maximises healthcare resources.

\section{ACKNOWLEDGEMENTS}

This project has been funded by the Inter Enterprise Computing Theme of the UK Department of Trade and Industry (DTI)-led Technology Programme, for which we are grateful. ECC and DRO are grateful for support by the Wales Office of Research and Development for Health and Social Care, Wales Assembly Government.

\section{REFERENCES}

Abidi, S.S.R, 2001, “An Intelligent Tele-Healthcare Environment Offering Person-Centric and WellnessMaintenance Services”, Journal of Medical Systems, Vol. 25, No.3.

Biometrics, a, date visited: July 2007, link visited: http://en.wikipedia.org/wiki/Biometrics

Blount, M., Batra, V.M., Capella, A.N., Ebling, M.R., Jerome, W.F., Martin, S. M., Nidd, M., and Wright, S.P., 2007, "Remote health-care monitoring using Personal Care Connect", Volume 46, InformationBased Medicine, IBM Systems Journal.

Conley, E.C., Owens, D.R. Luzio, S., Subramanian, M., Shaikh Ali, S., Hardisty, A., Rana, O. (2007) "Simultaneous Trend Analysis for Evaluating Outcomes in Patient-Centred Health Monitoring Services", Springer Journal of Healthcare Management Science, Submitted.

Clemensen, J., Larsen, S. B., and Bardram, J., 2004, "Developing Pervasive e-Health for Moving Experts from Hospital to Home", Proceedings of the IADIS eSociety Conference, pp.441-448, Avilla, Spain.

Common User Interface, June 2007, link visited: https://www.cui.nhs.uk/Pages/NHSCommonUserInterf ace.aspx

Continua Healthcare Alliance, June 2007, link visited: www.continuaalliance.org

Crossbow, June 2007, link visited: www.xbow.com

FIPS, 2002. "Security Requirements for Cryptographic Modules”, link accessed http://csrc.nist.gov/cryptval/140-2.htm

Gao, T., Massey, T., Sharp, J., Bishop, W., Bernstein, D., and Alm, A.., September 2006 "Integration of Triage and Biomedical Devices for Continuous, Real-Time, Automated Patient Monitoring". Proceedings of the IEEE Medical Devices and Biosensors Conference (IEEE MDBS 2006), Boston, MA..
Hein, A., Nee, O., Willemsen, D., Scheffold, T., Dogac, A., Laleci, G., 2006, “SAPHIRE - Intelligent Healthcare Monitoring based on Semantic Interoperability Platform - The Homecare Scenario", 1st European Conference on eHealth (ECEH'06), Fribourg, Switzerland.

Java ME: Mobile Information Device Profile (MIDP), date visited: July 2007, link visited: http://java.sun.com/products/midp/

JSR 82: Java APIs for Bluetooth, link visited: http://www.jcp.org/en/jsr/detail?id=82

King, H., Aubert, R., Herman, W., 1998, “Global burden of diabetes, 1995-2025: prevalence, numerical estimates, and projections" Journal: Diabetes Care,Volume: 21 Issue: 9, pp 1414-31.

Lorincz, K., Malan, M., Fulford-Jones, T., Nawoj, A., Clavel, A., Shnayder, V., Mainland, , S., Welsh, M., Oct-Dec 2004, "Sensor Networks for Emergency Response: Challenges and Opportunities”, In IEEE Pervasive Computing, Special Issue on Pervasive Computing for First Response.

Laszlo, date visited: July 2007, link visited: http://www.laszlosystems.com/.

ICP, Diabetes NSF (Wales) Standards, link accessed: www.wales.nhs.uk/sites3/home.cfm?orgid $=440$

Shnayder, V., Chen, B., Lorincz, K., Jones, T., \& Welsh, M., 2005, "Sensor Networks for Medical Care", Technical Report TR-08-05, Division of Engineering and Applied Sciences, Harvard University.

Shaikh-Ali, A., Rana, O.F. Hardisty, A., Subramanian, M., Luzio, S., Owens, D.R. and Conley, E.C., October 2007, "Portal Technologies for Patient-centred Integrated Care", European Conference on eHealth, Oldenburg, Germany.

SAPHE project, July 2007, link visited: http://ubimon.doc.ic.ac.uk/saphe/m338.html,

Subramanian, M., Shaikh-Ali, A., Rana, O.F. Hardisty, A. and Conley, E.C., October 2006, "Healthcare@Home: Research Models for atient-Centred Healthcare Services", John Vincent Atanasoff International Symposium on Modern Computing, Sofia, Bulgaria. IEEE Computer Society Press.

WHO, World Health Organisation, date visited: July 2007, link visited: http://www.who.int/en/

Wilson, C., Hicklin, R.A., Korves, H., Ulery, B., Zoepfl, M., Bone, M., Grother, P., Micheals, R., Otto, S., Watson, C., 2003, "Fingerprint Vendor Technology Evaluation”, National Institute of Standards and Technology, Mitretek Systems, NAVSEA Crane Division

Zigbee, date visited: July 2007, link visited: http://www.zigbee.org/en/index.asp 